\title{
From soft- to hard-sphere fluids: Crossover evidenced by high-frequency elastic moduli
}

\author{
Sergey Khrapak $\odot,{ }^{1,2,3,{ }^{*}}$ Nikita P. Kryuchkov, ${ }^{2}$ Lukiya A. Mistryukova, ${ }^{2}$ and Stanislav O. Yurchenko $\odot^{2, \dagger}$ \\ ${ }^{1}$ Joint Institute for High Temperatures, Russian Academy of Sciences, 125412 Moscow, Russia \\ ${ }^{2}$ Bauman Moscow State Technical University, 105005 Moscow, Russia \\ ${ }^{3}$ Institut für Materialphysik im Weltraum, Deutsches Zentrum für Luft- und Raumfahrt (DLR), 82234 Weßling, Germany
}

(Received 30 August 2020; accepted 27 April 2021; published 13 May 2021)

\begin{abstract}
The conventional (Zwanzig-Mountain) expressions for instantaneous elastic moduli of simple fluids predict their divergence as the limit of hard-sphere (HS) interaction is approached. However, elastic moduli of a true HS fluid are finite. Here we demonstrate that this paradox reveals the soft-to-hard-sphere crossover in fluid excitations and thermodynamics. With extensive in silico study of fluids with repulsive power-law interactions $\left(\propto r^{-n}\right)$, we locate the crossover at $n \simeq 10-20$ and develop a simple and accurate model for the HS regime. The results open prospects to deal with the elasticity and related phenomena in various systems, from simple fluids to melts and glasses.
\end{abstract}

DOI: 10.1103/PhysRevE.103.052117

\section{INTRODUCTION}

Understanding the mechanisms governing elastic moduli of substances is an important problem [1-5]: Elastic moduli are directly related to long-wavelength (sound) excitationsphonons-which play a crucial role in condensed matter, materials science, and soft matter.

For example, the celebrated Lindemann melting criterion states [6] that a three-dimensional (3D) solid melts when the vibrational amplitude of atoms around their equilibrium positions reaches about $\sim 0.1$ of the interatomic distance. Since the vibrational amplitude is dominated by long-wavelength excitations, the melting temperature can be expressed in terms of the shear and bulk moduli $[7,8]$. Another example is the Berezinskii-Kosterlitz-Thouless-Halperin-Nelson-Young (BKTHNY) theory of two-dimensional (2D) melting [9-14]: The condition for dislocation unbinding, responsible for crystal melting, can be expressed in terms of the (2D) shear and bulk moduli. Additionally, there is a possibility to formulate a 2D Lindemann-like criterion and relate it to the BKTHNY mechanism $[8,15]$. The instantaneous bulk and shear moduli are related to the $\alpha$ relaxation time in the framework of the shoving model, thus playing an important role in the physics of glass-forming liquids $[16,17]$. For instance, the temperature dependence of the shear viscosity can be expressed via the instantaneous shear modulus [18]. However, the effect of interaction softness on elastic moduli and collective excitations of fluids is still poorly understood.

The behavior of elastic moduli in systems with steeply repulsive interactions has remained a rather controversial issue for the last 50 years. The conventional (ZwanzigMountain) expressions for the high-frequency (instantaneous) bulk and shear moduli $[19,20]$ predict their divergence as the hard-sphere (HS) limit is approached from the side of soft interactions $[21,22]$. However, this divergence is inconsistent

\footnotetext{
*sergey.khrapak@gmx.de

†st.yurchenko@mail.ru
}

with other observations. The shear and bulk moduli of a true HS fluid are nonsingular and well defined [23], as well as elastic moduli of HS solids [24-25] and HS glass [26]. Finite values of the bulk modulus follow from finite isothermal and adiabatic sound velocities (evaluated from an appropriate equation of state) [27]. Finally, the finite shear modulus emerges from the analysis of transverse excitations in fluids [28].

The origin of this "paradoxical" situation has been attributed to the assumption of no structural relaxation upon density change $[29,30]$. The latter is well justified for soft interactions, but becomes unsuitable in the HS limit, because of an intrinsic length scale- the HS diameter. Hence, the divergence of the elastic moduli is artificial and the conventional expressions are just inappropriate in the HS limit. However, the question regarding where exactly the soft interaction stops to be soft enough to use the Zwanzig-Mountain expressions and how the HS limit is approached has remained obscure.

In this paper, we report on theoretical and extensive in silico studies of crossover from the soft-sphere (SSp) to the HS limit. Using molecular dynamics (MD) simulations, we consider a system of particles interacting via the inversepower-law (IPL) pair repulsion, $\varphi(r)=\epsilon(\sigma / r)^{n}$, where $\epsilon$ and $\sigma$ are the energy and length scales, and $n$ is the IPL exponent. With the methods developed in Ref. [31], we analyze in detail excitation spectra in dense IPL fluids with $n=8,12,20,50$, 100 , and 200 . We consistently compare the sound velocities obtained in silico and theoretically using the SSp and HS models, and discover that the crossover from the SSp to the HS dynamics occurs in the range $n \simeq 10-20$. For larger $n$ a simple theoretical approach is shown to provide good accuracy in estimating the elastic moduli.

\section{MD SIMULATIONS}

The equilibrium state of the IPL system is determined by a single reduced parameter [32], $\gamma_{n}=\rho \sigma^{3}(\epsilon / T)^{3 / n}$, where $\rho$ is the particle density and $T$ is the temperature (in energy units). 


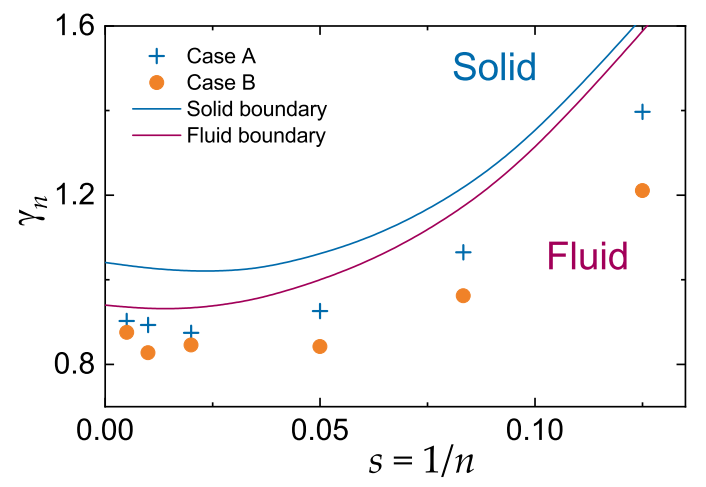

FIG. 1. Phase diagram of the IPL system: The fluid-solid coexistence region is bounded by the solid curves $[33,34]$; symbols denote the fluid state points studied in this work.

The phase diagram of the IPL family is sketched in Fig. 1, using available data [33,34]. In the HS limit, the phase state is determined by the packing fraction, $\eta=(\pi / 6) \rho \sigma^{3}$, with the freezing (melting) point at $\eta_{f} \simeq 0.494\left(\eta_{m} \simeq 0.545\right.$ ) [35]. The symbols in Fig. 1 (cases A and B) denote the state points studied in this work.

We performed MD simulations of $N=10648$ particles in the canonical $(N V T)$ ensemble with Nosé-Hoover thermostat and periodic boundary conditions in three dimensions. We used dimensionless units of energy, length, and mass $(\epsilon=1$, $\sigma=1$, and $m=1$ ), a cutoff radius $r_{c}=7.5$, and a numerical time step $\Delta t=5 \times 10^{-3} \sqrt{0.4 / T}$. All simulations were performed for $2 \times 10^{6}$ time steps, using the LAMMPS package [36]. The first $5 \times 10^{5}$ steps were used for equilibration and the following steps for the analysis.

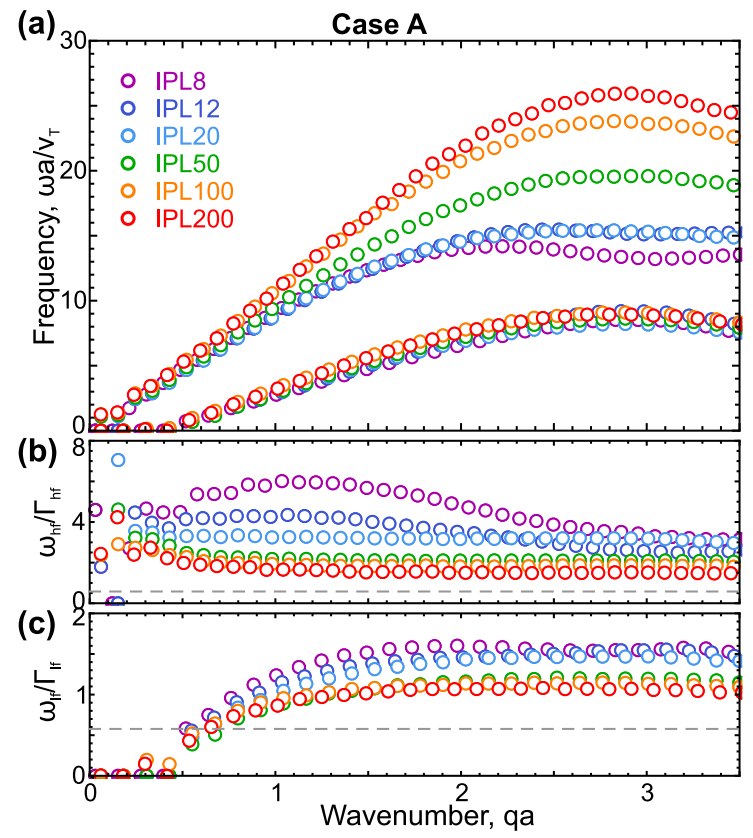

The excitation spectra were obtained using the procedures described in Refs. [31,37-40]. First, we calculated the velocity current spectra $[31,41]$ :

$$
C_{L, T}(\mathbf{q}, \omega)=\int d t e^{i \omega t} \operatorname{Re}\left\langle j_{L, T}(\mathbf{q}, t) j_{L, T}(-\mathbf{q}, 0)\right\rangle,
$$

where $\mathbf{q}$ and $\omega$ are the wave vector and the frequency; $\mathbf{j}_{L}=\mathbf{q}(\mathbf{q} \cdot \mathbf{j}) / q^{2}$ and $\mathbf{j}_{T}=\mathbf{j}-\mathbf{j}_{L}$ are the longitudinal and transverse components of the current $\mathbf{j}(\mathbf{q}, t)=$ $N^{-1} \sum_{s} \mathbf{v}_{s}(t) \exp \left(i \mathbf{q} \mathbf{r}_{s}(t)\right)$; and $\mathbf{v}_{s}(t)=\dot{\mathbf{r}}_{s}(t)$ is the velocity of the $s$ th particle. In isotropic fluids the directional dependence vanishes: $C_{L, T}(\mathbf{q}, \omega) \equiv C_{L, T}(q, \omega)$. The total current spectra $C(q, \omega)=C_{L}(q, \omega)+2 C_{T}(q, \omega)$ were fitted at each $q$ value with the two-oscillator model [31]:

$$
\begin{aligned}
C(q, \omega) \propto & \frac{\Gamma_{\mathrm{hf}}}{\left(\omega-\omega_{\mathrm{hf}}\right)^{2}+\Gamma_{\mathrm{hf}}^{2}}+\frac{\Gamma_{\mathrm{hf}}}{\left(\omega+\omega_{\mathrm{hf}}\right)^{2}+\Gamma_{\mathrm{hf}}^{2}} \\
& +\frac{2 \Gamma_{\mathrm{lf}}}{\left(\omega-\omega_{\mathrm{lf}}\right)^{2}+\Gamma_{\mathrm{lf}}^{2}}+\frac{2 \Gamma_{\mathrm{lf}}}{\left(\omega+\omega_{\mathrm{lf}}\right)^{2}+\Gamma_{\mathrm{lf}}^{2}},
\end{aligned}
$$

where $\omega_{\mathrm{hf}, \text {,ff }}$ and $\Gamma_{\mathrm{hf}, \text { If }}$ are the frequencies and damping rates of the high- and low-frequency branches.

\section{RESULTS}

The excitation spectra in fluids are shown in Figs. 2(a)2(c) and 2(d)-2(f), for the state points of cases A and B in Fig. 1. The low- and high-frequency dispersion curves shown in Figs. 2(a) and 2(d) behave similarly to those reported in Refs. [31,38-40]. In the long-wavelength limit, as usual, these branches are attributed to the longitudinal and transverse collective modes $[31,40]$. Remarkably, the branch $\omega_{\text {lf }}$ behaves quasiuniversally (in reduced units), being almost independent

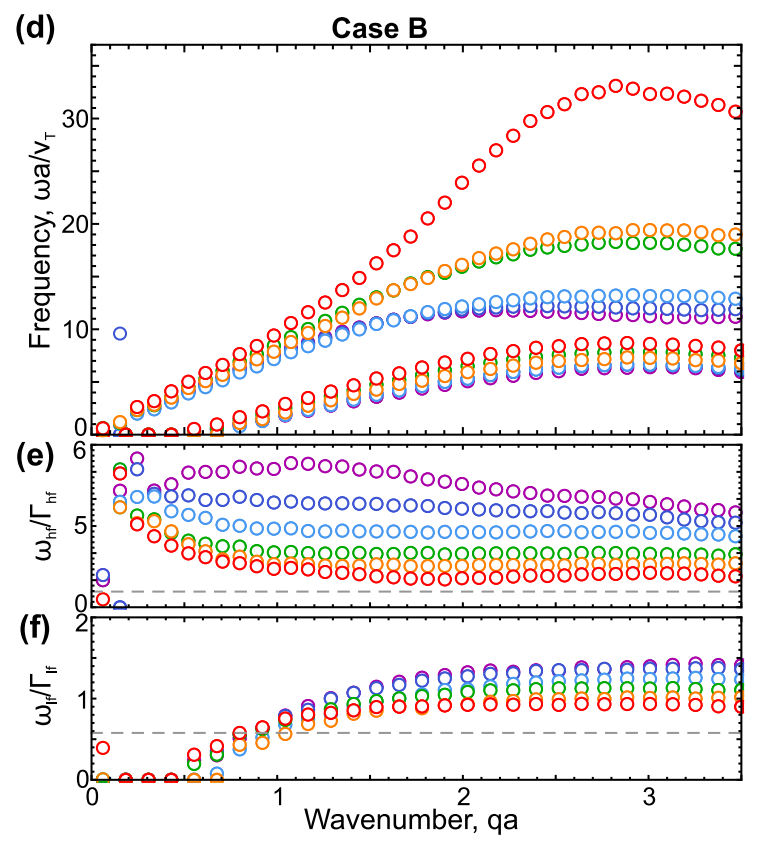

FIG. 2. Collective excitation spectra in dense IPL fluids: The results are shown for state points of (a-c) case A and (d-f) case B in Fig. 1 . The high- and low-frequency branches in (a) and (d) correspond to the longitudinal and transverse modes in the long-wavelength limit. Here $v_{T}=\sqrt{T / m}$ is the thermal velocity and $a=(4 \pi \rho / 3)^{-1 / 3}$ is the Wigner-Seitz radius. Panels (b) and (c) and panels (e) and (f) show the ratios of the oscillation frequency to the damping rate for the high- and low-frequency branches, respectively. Below the grey dashed lines $\omega / \Gamma=1 / \sqrt{3}$, the branches become overdamped [31]. 
of the IPL exponent, in contrast to $\omega_{\mathrm{hf}}$. Here, the reduced maximum frequency increases with $n$, demonstrating clear positive sound dispersion for $n \gtrsim 20$.

In Figs. 2(b), 2(c), 2(e), and 2(f), the ratios $\omega / \Gamma$ of the real frequency to the damping rate for the high- and lowfrequency branches are shown to illustrate the quality factor of the oscillations. The horizontal dashed lines correspond to $\omega / \Gamma=1 / \sqrt{3}$, below which even oscillating modes with $\omega \neq$ 0 become overdamped [31,37]; $\omega / \Gamma=0$ corresponds to the gapped excitations of the low-frequency (transverse) branch. The ratio $\omega / \Gamma$ grows monotonically for transverse excitations in the intermediate $q$ regime and is systematically larger for softer potentials (smaller $n$ ). On the contrary, the factor $\omega / \Gamma$ of the high-frequency branch has a maximum in the vicinity of $q a \simeq 1.1$ in the SSp regime (the position of the maximum correlates with the transition from the hydrodynamic regime to the limit of individual particles [31]), but drops monotonously with $q a$ in the HS regime $(n \gtrsim 20)$. The qualitative changes in the excitation spectra at $n \simeq 20$, highlighted in Fig. 2, point to a crossover from soft- to hard-sphere fluid collective dynamics.

To analyze this crossover in detail, consider the longwavelength excitations in Fig. 2. The longitudinal (bulk) and transverse (shear) sound velocities are evaluated from

$$
c_{s}=\lim _{q \rightarrow 0} \frac{\partial \omega_{\mathrm{hf}}}{\partial q}, \quad c_{t}=\lim _{q \rightarrow q_{g}+0} \frac{\partial \omega_{\mathrm{lf}}}{\partial q},
$$

where $c_{t}$ is calculated near the gap in reciprocal space ( $q$ gap), corresponding to the minimum wave number $q_{g}$, below which $\omega_{\mathrm{lf}}=0$, and where typically $\omega_{\mathrm{lf}} \simeq c_{t}\left(q-q_{g}\right)$ [31]. With the linear fitting (3) of the MD excitation spectra (Fig. 2), we have obtained the sound velocities.

The sound velocities deduced from MD simulation contain information about the instantaneous bulk and shear moduli [29] via the relations $K_{\infty}=m \rho c_{s}^{2}$ and $G_{\infty}=m \rho c_{t}^{2}$, respectively. For repulsive interactions, including the HS limit, the instantaneous and adiabatic moduli are numerically close $[29,30,42]$, and we do not distinguish between them in the following. In the conventional SSp paradigm, the moduli are expressed via the pair potential $\varphi(r)$ and the radial distribution function (RDF) $g(r)[19,20]$ :

$$
\begin{aligned}
G_{\infty} & =\rho T+\frac{2 \pi \rho^{2}}{15} \int_{0}^{\infty} d r r^{3} g(r)\left[r \varphi^{\prime \prime}(r)+4 \varphi^{\prime}(r)\right], \\
K_{\infty} & =\frac{5}{3} \rho T+\frac{2 \pi \rho^{2}}{9} \int_{0}^{\infty} d r r^{3} g(r)\left[r \varphi^{\prime \prime}(r)-2 \varphi^{\prime}(r)\right] .
\end{aligned}
$$

The first terms in expressions for $G_{\infty}$ and $K_{\infty}$ correspond to the kinetic (ideal gas) contribution, while the second ones are the configurational (excess) contribution, $G_{\mathrm{ex}}$ and $K_{\mathrm{ex}}$, which are dominant in dense fluids. In IPL fluids $G_{\text {ex }}$ and $K_{\text {ex }}$ are directly related to the excess pressure $P_{\mathrm{ex}}$ as $G_{\mathrm{ex}}=(n-3) P_{\mathrm{ex}} / 5$ and $K_{\mathrm{ex}}=(n+3) P_{\mathrm{ex}} / 3$.

The paradoxical divergence of elastic moduli now becomes particularly clear: As $n$ increases, $G_{\text {ex }}$ and $K_{\text {ex }}$ diverge as $\propto n$, because $P_{\mathrm{ex}}$ remains finite in the HS limit. The spectra in Fig. 2, however, evidence that this is not the case and the actual elastic moduli $\left(\propto c_{s}^{2}\right.$ and $\left.c_{t}^{2}\right)$ are finite. This proves that the SSp expressions (4) become unsuitable at large $n$. But where exactly do soft spheres become not so soft any more?
To answer the question, we have constructed a simple HS asymptotic model for elastic moduli at large $n$. We start with the Carnahan-Starling (CS) equation of state [43] of the HS fluid. The pressure is written as

$$
P(\rho, T)=\rho T Z(\eta), \quad Z(\eta)=\frac{1+\eta+\eta^{2}-\eta^{3}}{(1-\eta)^{3}},
$$

where $Z(\eta)$ is the CS compressibility, and the effective HS packing fraction $\eta$ depends on the effective HS diameter $d_{\text {eff }}$ specified below. The adiabatic bulk modulus $K_{s}$ follows straightforwardly from the factor $Z[27,44]$ :

$$
K_{s}=\rho T\left[Z(\eta)+\eta d Z(\eta) / d \eta+\frac{2}{3} Z^{2}(\eta)\right] .
$$

For the shear modulus, we combine the expression for $G_{\infty}$ derived by Miller [23,30] with the approximation for $g^{\prime}(1)$ reported in Ref. [45]:

$$
G_{\infty}=\rho T\left[1-\frac{8}{5} \eta g^{\prime}(1)\right], \quad g^{\prime}(1)=-\frac{9 \eta(1+\eta)}{2(1-\eta)^{4}},
$$

where $g^{\prime}(1)$ denotes the reduced derivative at contact, $g^{\prime}(1)=$ $\lim _{\epsilon \rightarrow 0}[d g(x) / d x]_{x=1+\epsilon}$ with $x=r / d$.

The final step is to determine the effective HS diameter $d_{\text {eff }}$. Several approximations have been proposed over the years; among the most familiar are those by Rowlinson, Barker and Henderson, and Stillinger [46-49]. For the IPL potential they result in the generic condition

$$
d_{\mathrm{eff}}=\mathcal{A} \sigma(\epsilon / T)^{1 / n},
$$

where $\mathcal{A}$ is a numerical factor, which tends to unity as $n \rightarrow$ $\infty$, but appears approximation dependent at finite $n$. In the considered case the Rowlinson and Barker and Henderson (RBH) approximations coincide and we get $\mathcal{A}=\Gamma(1-1 / n)$. The Stillinger approximation yields $\mathcal{A}=(1 / \ln 2)^{1 / n}$. Yet another approximation, which appears particularly suitable for the problem at hand, results from equating the potential interaction energy at $d_{\text {eff }}$ to the average kinetic energy $3 T / 2$,

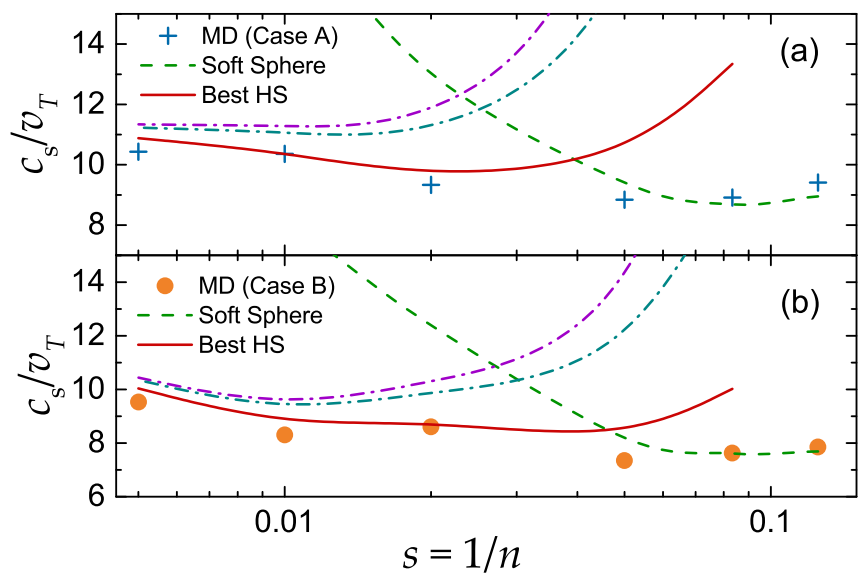

FIG. 3. Reduced longitudinal sound velocity $c_{s} / v_{\mathrm{T}}$ versus the softness parameter $s=1 / n$ of the IPL fluids for (a) case A and (b) case B. Symbols correspond to results from MD simulation. The dashed and solid curves correspond to the SSp and the best HS asymptote, Eqs. (4) and (6), respectively. Two additional dash-dotted curves in the HS regime correspond to the effective HS diameters estimated using RBH (upper) and Stillinger (lower) approaches. 


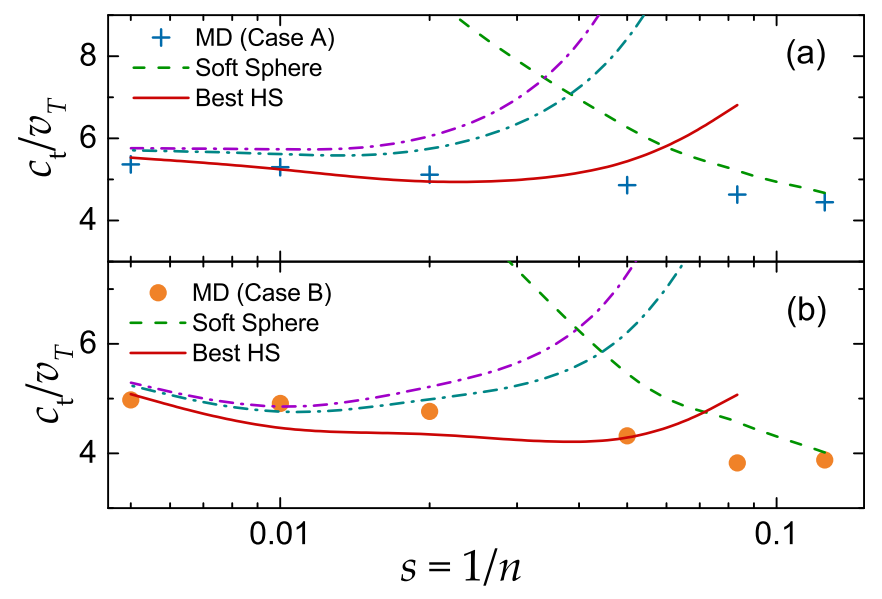

FIG. 4. Reduced transverse sound velocity $c_{t} / v_{\mathrm{T}}$ versus the softness parameter of the IPL fluid. The HS asymptotes are calculated using Eq. (7). Other notation is the same as in Fig. 3.

resulting in $\mathcal{A}=(2 / 3)^{1 / n}$. For a given effective HS diameter, the sound velocities are evaluated from $c_{s}=\sqrt{K_{s} / \rho m}$ and $c_{t}=\sqrt{G_{\infty} / \rho m}$ using Eqs. (6) and (7).

The theoretical and MD results are compared in Figs. 3 and 4 , showing our main result. Here, the sound velocities expressed in units of the thermal velocity $v_{\mathrm{T}}=\sqrt{T / m}$, are plotted. The dashed curves correspond to SSp (ZwanzigMountain) expressions, illustrating their range of suitability. The SSp description fails at $n \simeq 20$, if judged from $c_{s}$ in Fig. 3 and at $n \simeq 10$ if considering $c_{t}$ in Fig. 4. The red solid curves correspond to the HS asymptote with the effective HS diameter evaluated from Eq. (8) with $\mathcal{A}=(2 / 3)^{1 / n}$ (other approaches converge to the same result at $n \rightarrow \infty$, but somewhat overestimate elastic moduli at finite $n$ ).

\section{DISCUSSION}

Our results can be useful to better understand a broad range of previous studies and to suggest future research directions, and are not limited to modeling IPL fluids. In dense fluids, the motion of an atom is dominated by repulsion from its nearest neighbors. Hence, the system properties are mainly governed by the shape of the interaction potential in a relatively narrow range of distances, near the average interparticle separation [50,51]. In this region, the (extended) IPL potential can accurately fit the actual potential [52]. The fit defines an effective IPL exponent $n_{\text {eff }}$, regulating the effective softness of the actual interaction.

For example, the typical effective IPL exponent for the Lennard-Jones (LJ) potential, describing liquified noble gases, is $n_{\text {eff }} \simeq 18$ (at moderate densities) [53]: This might appear to be surprising, but the reason is that the attractive $\left(\propto r^{-6}\right)$ term of the $\mathrm{LJ}$ potential makes its repulsive short-range branch considerably steeper than just the $\propto r^{-12}$ repulsive term. Only at high densities does $n_{\text {eff }}$ approach 12 [52]. This should be kept in mind when analyzing instantaneous elastic moduli (in particular, the shear modulus) in liquified noble gases [54]. The same obviously applies to generalized LJ $m-n$ potentials, in particular at $m>12$.

In many liquid metals, the interactions can be approximated by the IPL potential. Extensive density functional theory (DFT) calculations of 58 liquid elements at their triple points demonstrate that most metallic elements exhibit strong correlations between virial and potential energy and thus obey "hidden scale invariance" even at these relatively low densities [55]. The structure and phase diagrams of many such elements are consistent with the IPL model. Typical DFT computed density scaling exponents yield $n_{\text {eff }} \lesssim 10$. However, in some cases $n_{\text {eff }}$ are quite large. For instance, for $\mathrm{Rh}, \mathrm{Cd}$, Os, Ir, and Pt we observe $n_{\text {eff }} \gtrsim 15$ with an extreme value $n_{\text {eff }} \simeq 23.7$ for Au [55]. A careful account of elastic properties of these systems is warranted.

Recently, a microscopic model for the temperature dependence of the shear viscosity and fragile-strong behavior of liquid metals in the supercooled regime has been developed by combining the shoving model with the SSp expression for $G_{\infty}$ [18]. The repulsion steepness of the interaction potential has emerged as the crucial parameter governing the glass fragility, which has been shown to increase monotonously with the repulsion steepness. This result is heavily based on the SSp expression for $G_{\infty}$, and can be naturally tested with a more appropriate expression for $G_{\infty}$ in the regime of steep HS-like interactions.

\section{CONCLUSION}

To conclude, the comprehensive analysis of different approximations for classical liquids, considered from macroscopic (elastic properties) and microscopic (excitation spectra) points of view, clearly reveals when soft spheres become hard spheres. The results have allowed us to unravel the paradoxical divergences of classical Zwanzig-Mountain formulas and to determine the range of their suitability, providing a useful input for future studies of fluids and glasses, from atomic to macromolecular systems.

\section{ACKNOWLEDGMENTS}

We would like to thank V. Nosenko and M. Schwabe for careful reading of the manuscript. N.P.K. and S.O.Y. are grateful to BMSTU State Assignment for infrastructural support. S.O.Y. acknowledges Acad. V.V. Brazhkin for his interest to the work and fruitful discussions about where soft spheres become hard. Analysis of the effect of interaction steepness on collective modes in fluids was supported by the Russian Science Foundation, Grant No. 20-12-00356.
[1] T. G. Mason and D. A. Weitz, Optical Measurements of Frequency-Dependent Linear Viscoelastic Moduli of Complex Fluids, Phys. Rev. Lett. 74, 1250 (1995).
[2] T. Kokubo, H.-M. Kim, and M. Kawashita, Novel bioactive materials with different mechanical properties, Biomaterials $\mathbf{2 4}$, 2161 (2003). 
[3] T. G. Mason, Estimating the viscoelastic moduli of complex fluids using the generalized Stokes-Einstein equation, Rheol. Acta 39, 371 (2000).

[4] J. C. Dyre, Colloquium: The glass transition and elastic models of glass-forming liquids, Rev. Mod. Phys. 78, 953 (2006).

[5] S. V. Nemilov, Interrelation between shear modulus and the molecular parameters of viscous flow for glass forming liquids, J. Non-Cryst. Solids 352, 2715 (2006).

[6] F. Lindemann, The calculation of molecular vibration frequencies, Z. Phys. 11, 609 (1910).

[7] U. Buchenau, R. Zorn, and M. A. Ramos, Probing cooperative liquid dynamics with the mean square displacement, Phys. Rev. E 90, 042312 (2014).

[8] S. A. Khrapak, Lindemann melting criterion in two dimensions, Phys. Rev. Res. 2, 012040(R) (2020).

[9] J. M. Kosterlitz and D. J. Thouless, Ordering, metastability and phase transitions in two-dimensional systems, J. Phys. C 6, 1181 (1973).

[10] D. R. Nelson and B. I. Halperin, Dislocation-mediated melting in two dimensions, Phys. Rev. B 19, 2457 (1979).

[11] A. P. Young, Melting and the vector coulomb gas in two dimensions, Phys. Rev. B 19, 1855 (1979).

[12] V. L. Berezinskii, Destruction of long-range order in onedimensional and 2-dimensional systems having a continuous symmetry group 1-classical systems, J. Exp. Theor. Phys. 32, 493 (1971).

[13] J. M. Kosterlitz, Nobel lecture: Topological defects and phase transitions, Rev. Mod. Phys. 89, 040501 (2017).

[14] V. N. Ryzhov, E. E. Tareyeva, Yu. D. Fomin, and E. N. Tsiok, Berezinskii-Kosterlitz-Thouless transition and two-dimensional melting, Phys.-Usp. 60, 857 (2017).

[15] S. Khrapak, Note: Melting criterion for soft particle systems in two dimensions, J. Chem. Phys. 148, 146101 (2018).

[16] J. C. Dyre and N. B. Olsen, Landscape equivalent of the shoving model, Phys. Rev. E 69, 042501 (2004).

[17] J. C. Dyre and W. H. Wang, The instantaneous shear modulus in the shoving model, J. Chem. Phys. 136, 224108 (2012).

[18] G. Chevallard, K. Samwer, and A. Zaccone, Atomic-scale expressions for viscosity and fragile-strong behavior in metal alloys based on the Zwanzig-Mountain formula, Phys. Rev. Res. 2, 033134 (2020).

[19] R. Zwanzig and R. D. Mountain, High-frequency elastic moduli of simple fluids, J. Chem. Phys. 43, 4464 (1965).

[20] P Schofield, Wavelength-dependent fluctuations in classical fluids: I. The long wavelength limit, Proc. Phys. Soc. 88, 149 (1966).

[21] H. L. Frisch, High frequency linear response of classical fluids, Physics 2, 209 (1966).

[22] D. M. Heyes and P. J. Aston, Elastic moduli of simple fluids with steeply repulsive potentials, J. Chem. Phys. 100, 2149 (1994).

[23] B. N. Miller, Elastic moduli of a fluid of rigid spheres, J. Chem. Phys. 50, 2733 (1969).

[24] D. Frenkel and Anthony J. C. Ladd, Elastic Constants of HardSphere Crystals, Phys. Rev. Lett. 59, 1169 (1987).

[25] B. B. Laird, Weighted-density-functional theory calculation of elastic constants for face-centered-cubic and body-centeredcubic hard-sphere crystals, J. Chem. Phys. 97, 2699 (1992).

[26] H Löwen, Elastic constants of the hard-sphere glass: A density functional approach, J. Phys.: Condens. Matter 2, 8477 (1990).
[27] Y. Rosenfeld, Sound velocity in liquid metals and the hard-sphere model, J. Phys.: Condens. Matter 11, L71 (1999).

[28] T. Bryk, A. Huerta, V. Hordiichuk, and A. D. Trokhymchuk, Non-hydrodynamic transverse collective excitations in hardsphere fluids, J. Chem. Phys. 147, 064509 (2017).

[29] S. Khrapak, B. Klumov, and L. Couedel, Collective modes in simple melts: Transition from soft spheres to the hard sphere limit, Sci. Rep. 7, 7985 (2017).

[30] S. Khrapak, Elastic properties of dense hard-sphere fluids, Phys. Rev. E 100, 032138 (2019).

[31] N. P. Kryuchkov, L. A. Mistryukova, V. V. Brazhkin, and S. O. Yurchenko, Excitation spectra in fluids: How to analyze them properly, Sci. Rep. 9, 10483 (2019).

[32] D. H. E. Dubin and H. Dewitt, Polymorphic phase transition for inverse-power-potential crystals keeping the first-order anharmonic correction to the free energy, Phys. Rev. B 49, 3043 (1994).

[33] R. Agrawal and D. A. Kofke, Solid-Fluid Coexistence for Inverse-Power Potentials, Phys. Rev. Lett. 74, 122 (1995).

[34] R. Agrawal and D. A. Kofke, Thermodynamic and structural properties of model systems at solid-fluid coexistence, Mol. Phys. 85, 23 (1995).

[35] L. Berthier and G. Biroli, Theoretical perspective on the glass transition and amorphous materials, Rev. Mod. Phys. 83, 587 (2011).

[36] S. Plimpton, Fast parallel algorithms for short-range molecular dynamics, J. Comput. Phys. 117, 1 (1995).

[37] S. O. Yurchenko, K. A. Komarov, N. P. Kryuchkov, K. I. Zaytsev, and V. V. Brazhkin, Bizarre behavior of heat capacity in crystals due to interplay between two types of anharmonicities, J. Chem. Phys. 148, 134508 (2018).

[38] N. P. Kryuchkov, V. V. Brazhkin, and S. O. Yurchenko, Anticrossing of longitudinal and transverse modes in simple fluids, J. Phys. Chem. Lett. 10, 4470 (2019).

[39] E. V. Yakovlev, N. P. Kryuchkov, P. V. Ovcharov, A. V. Sapelkin, V. V. Brazhkin, and S. O. Yurchenko, Direct experimental evidence of longitudinal and transverse mode hybridization and anticrossing in simple model fluids, J. Phys. Chem. Lett. 11, 1370 (2020).

[40] N. P. Kryuchkov, L. A. Mistryukova, A. V. Sapelkin, V. V. Brazhkin, and S. O. Yurchenko, Universal Effect of Excitation Dispersion on the Heat Capacity and Gapped States in Fluids, Phys. Rev. Lett. 125, 125501 (2020).

[41] J. P. Hansen and I. R. McDonald, Theory of Simple Liquids (Elsevier, London, 2006).

[42] S. A. Khrapak, Relations between the longitudinal and transverse sound velocities in strongly coupled Yukawa fluids, Phys. Plasmas 23, 024504 (2016).

[43] N. F. Carnahan and K. E. Starling, Equation of state for nonattracting rigid spheres, J. Chem. Phys. 51, 635 (1969).

[44] S. A. Khrapak, Note: Sound velocity of a soft sphere model near the fluid-solid phase transition, J. Chem. Phys. 144, 126101 (2016).

[45] F.-M. Tao, Y. Song, and E. A. Mason, Derivative of the hardsphere radial distribution function at contact, Phys. Rev. A 46, 8007 (1992).

[46] J. S. Rowlinson, The statistical mechanics of systems with steep intermolecular potentials, Mol. Phys. 8, 107 (1964). 
[47] J. A. Barker and D. Henderson, Perturbation theory and equation of state for fluids. II. a successful theory of liquids, J. Chem. Phys. 47, 4714 (1967).

[48] D. Henderson, Rowlinson's concept of an effective hard sphere diameter, J. Chem. Eng. Data 55, 4507 (2010).

[49] F. H. Stillinger, Phase transitions in the gaussian core system, J. Chem. Phys. 65, 3968 (1976).

[50] S. A. Khrapak, M. Chaudhuri, and G. E. Morfill, Communication: Universality of the melting curves for a wide range of interaction potentials, J. Chem. Phys. 134, 241101 (2011).

[51] L. Bøhling, A. A. Veldhorst, T. S. Ingebrigtsen, N. P. Bailey, J. S. Hansen, S. Toxvaerd, T. B. Schrøder, and J. C. Dyre, Do the repulsive and attractive pair forces play separate roles for the physics of liquids? J. Phys.: Condens. Matter 25, 032101 (2013).

[52] L. Bøhling, N. P. Bailey, T. B. Schrøder, and J. C. Dyre, Estimating the density-scaling exponent of a monatomic liquid from its pair potential, J. Chem. Phys. 140, 124510 (2014).

[53] U. R. Pedersen, N. P. Bailey, T. B. Schrøder, and J. C. Dyre, Strong Pressure-Energy Correlations in van der Waals Liquids, Phys. Rev. Lett. 100, 015701 (2008).

[54] S. A. Khrapak, Sound velocities of Lennard-Jones systems near the liquid-solid phase transition, Molecules 25, 3498 (2020).

[55] F. Hummel, G. Kresse, J. C. Dyre, and U. R. Pedersen, Hidden scale invariance of metals, Phys. Rev. B 92, 174116 (2015). 